\title{
Modified WFRFT-based Transform Domain Communication System I ncorporating with Spectrum Mismatching
}

\author{
Ruiyang $\mathrm{Xu}^{1}$, Xinyu $\mathrm{Da}^{1}$ Yuan Liang ${ }^{1}$ and Hang $\mathrm{Hu}^{1}$ \\ ${ }^{1}$ Information and Navigation College, Air Force Engineering University \\ Shannxi, Xi‘an, China \\ [e-mail: lixueyuanxuruiyang@126.com] \\ *Corresponding author: Ruiyang $\mathrm{Xu}$
}

Received November 29, 2017; revised March 15, 2018; revised April 12, 2018; accepted April 26, 2018; published October 31, 2018

\begin{abstract}
The transform domain communication system (Hereinafter referred to as TDCS) takes on numerous advantages, inclusive of anti-jamming and low probability of detection. Yet its application is confined by the consistent spectrum in the transmitter and receiver, which is not possible in the case of a huge distance exsits between them. In this paper, a TDCS based modified weighted fractional fourier transform (WFRFT) is proposed to solve the problem resulting from spectrum mismatching for TDCS application. The amplitude and phase information are incorporated with the TDCS signals and transmit to the receiver together in the wake of a modified WFRFT. The basic function and the TDCS signals shall be accessible to the receivers in the wake of an inverse WFRFT transform, which make sure that the original information can be demodulated properly. The system's reliability while transmitting signals with different modulation methods and with spectrum mismatching is demonstrated by bit error rate (BER). In the meantime, the constellations of the signals and the BER performances at the eavesdropper demonstrate the proposed system is better secured.
\end{abstract}

Keywords: Modified WFRFT, Transform Domain Communication System (TDCS), Spectrum Mismatched

This research was supported by a research grant from the National Natural Science Fundation of China under Grant 61571460 , 


\section{Introduction}

Transform domain communication system (TDCS) counts as a wireless communication system developing on the basis of cognitive radio (CR), transmitting spectrum communication and transform domain processing technique [1][2], capable of taking on good performance in anti-interference and anti-interception, as well as multi-access communication. These advantages of this technique have aroused extensive concerns in recent years. An unique sequence and a special cyclic code shifting key are designed to establish a novel framework of TDCS-based cognitive radio networks to avoid multiuser interference [3]. The preamble comprising multiple identical training sequence is presented to simplify the estimation of symbol timing offset and carrier frequency offset to solve the synchronization in TDCS [4]. Magnitude weights selected by the modified backtracking search optimization algorithm are adopted to cope with the high peak-to-average power ratio of TDCS signals [5]. A method of extending constellation by incorporating multidimensional vectors [6] and an unified modulation framework encompassing the previously reported modulation schemes under one framework [7] are proposed to elevate the spectrum efficiency of TDCS. In the TDCS, the design of the basic function is deemed as the crux to attain the system abilities. The anti-jamming capability of the TDCS can be optimized by analyzing the short-time spectrum characteristic of single-chip waveform coded basic function[8]. A double-threshold basic function is designed to solve the problems that the single-threshold basic function is fixed in design, and the system output is not factored in [9].

Following the foregoing references, it can be deduced that the basic function is of crucial significance for TDCS. Yet the basic function is the scalar product of a complex pseudo random vector and the amplitude spectrum, which is acquired through sensing the occupied spectrum at the transmitter and at the receiver, respectively. The difference in spectrum at the transmitter and the receiver (e.g. they are markedly separated) shall result in differences of the basic function. Consequently the information reception shall be affected, and the performance of the system shall also be affected. One method to solve this problem is to build a public channel in the CR network scenario to exchange the spectrum sensing results between the transmitter and the receiver [10][11], whereas it shall accordingly make the system more complicated and give rise to new security problem. The basic function shall also be interfered by the noise and fading in the channel, which shall impact the system's reliability. Equal-gain combining method can be adopted to design the basic function at the receiver to ensure the coherence of the basic function [12]. Yet this method requires the phase information of the signal, which confines the application of this system.

To solve the foregoing problems, a TDCS is proposed for spectrum mismatching scenarios in the light of a modified WFRFT. WFRFT is a novel time-frequency analyzing tool developed from the fourier transform (FT) and the fractional fourier transform (FRFT). Since it inherits and improves the character of FRFT [13], WFRFT has aroused the concerns from communication field progressively in recent years by virtue of its special characters. The distinctive weighted combination structure and evident variation in the statistics properties make it perform better in physical layer security [14]. The WFRFT-based communication system is also a hybrid carrier (HC) system [15], which takes on the advantages of both the single carrier (SC) system and the multi-carrier (MC) system [16][17], and can be extended to a HC-CDMA system [18]. The WFRFT can be adopted as a precoding scheme in the communication system to outperform the original system in some areas [19][20]. Accordingly 
the communication system can be safer whereby cooperative method [21], constellation scrambling method [22] and parallel combinatory method [23]. Except the applications mentioned above, WFRFT is also an effective method in physical layer security, especially after combining with polarization modulation [24][25], which enhances the information safty of dual-polarized satellite systems and demonstrates better performance than the same system without it [26].

As TDCS takes on a good performance and WFRFT has special characters, a modified WFRFT-based TDCS is proposed in this paper under the condition that the spectrum is mismatching at the transmitter and the receiver. The contribution of this paper can be summarized as follow: To transmit the information of the spectrum at the transmitter, a modified WFRFT is adopted to transmit different signals at the same time, which makes it convenient to transmit the basic function together with the signals in TDCS without building a new public channel. The security of the signal is also been optimized by virtue of the character of WFRFT. In the meantime, the system's reliability when transmitting different modulated signals and its comparisons between the traditional TDCS and the method proposed in reference [12] are anatomized. Moreover, the theoretical BER equation is derived to offer a standard for justifying the reliability of the system. The security performance of the system is also been anatomized through drawing the comparison between the constellation generated by different $\alpha$ and the BER at the legal receiver and the eavesdropper.

The rest of this paper is organized as follows: The basic knowledge about the TDCS and spectrum mismatching is presented in Section 2. The WFRFT and its modification are presented in Section 3. The system model of the modified WFRFT-based TDCS is defined and the theoretical BER equation is derived in Section 4. The signal character and the system performance is anatomized in Section 5. Eventually, Section 6 draws the conclusion.

\section{Transform Domain Communication System}

The block diagrams of both transmitter and receiver of a traditional TDCS are exhibited in Fig. 1. The spectral information $A_{0 k}(\omega)$ is estimated through sampling the local electromagnetic environment. On that basis an amplitude shaping process is undertaken to output $A_{k}(\omega)$, which encompasses zeros and ones. Spectral regions surmounting the threshold are assigned a value of zero and other regions are assigned a value of one. A pseudorandom phase is generated whereby a pseudorandom sequence with the form of $e^{j \theta_{k}}$ through a process called phase mapping. $\theta_{k}$ is selected from phase mapper, which is constituted by $2^{r}$ phase points. Additionally, the points are uniformly distributed in $[0,2 \pi)$ [27]. i.e.

$$
\theta_{k} \in\left[0, \frac{2 \pi}{2^{r}}, \frac{4 \pi}{2^{r}}, \ldots, \frac{2 \pi\left(2^{r}-1\right)}{2^{r}}\right] \quad k=1,2, \ldots, N
$$

where $k$ is the index of the chosen phase and $r$ is the total number of the numbers chosen from a pseudorandom sequence, which are applied to generate the $2^{r}$ phase points.

The resulting PR phase is multiplied element-by-element with $A_{k}(\omega)$ to generate the basic vector. The spectral basic vector is amplitude-scaled to ensure constant energy between symbols and processed by an inverse fourier transform to generate a noise-like time domain basic function (BF) $b_{n}$. The form of $\mathrm{BF}$ is defined as: 


$$
b_{n}=\frac{1}{N} \sum_{k=0}^{N-1} A_{k}(\omega) e^{j \theta_{k}} e^{\frac{j 2 \pi k n}{N}} n=0,1, \ldots, N-1
$$

The BF is stored and employed to modulate the data $d$. For TDCS effectuation, cyclic shifting keying modulation (CSK) is the most frequently adopted modulation method, comprising binary cyclic shifting keying (BCSK), cyclic code-shifting keying (CCSK), etc. These kinds of modulation methods provide low cross-correlation between communication symbols. The signal after modulation shall be:

$$
S_{n}=\frac{1}{N} \sum_{k=0}^{N-1} A_{k} e^{j \theta_{k}} e^{\frac{j 2 \pi k n}{N}} d \quad n=0,1, \ldots, N-1
$$

A locally generated basic function is required to be adequately acquired and accurately aligned by the TDCS receiver through estimating the local spectral information prior to the signal demodulation. Assuming a transmitter-receiver pair observes identical spectral, they jointly, yet independently, notch out identical spectral regions during BF generation. Assuming the $\mathrm{BF}$ generated by the transmitter and the receiver are $b_{n}$ and $b_{n}{ }^{\prime}$ respectively,where $b_{n}{ }^{\prime}$ is defined correspondingly as:

$$
b_{n}{ }^{\prime}=\frac{1}{N} \sum_{k=0}^{N-1} A^{\prime}{ }_{k}(\omega) e^{j \theta_{k}^{\prime}} e^{\frac{j 2 \pi k n}{N}} \quad n=0,1, \ldots, N-1
$$

In the wake of completing signal synchronization, the TDCS receiver may proceed with demodulation and the result is adopted to output the correspond symbol of the demodulation information. The signal before decision can be denoted as:

$$
y=\sum_{n=0}^{N-1}\left(s_{n}+n\right)\left(b_{n}^{\prime}\right)^{*}=\sum_{k=0}^{N-1} A_{k} A_{k}^{\prime} e^{j\left(\theta_{k}-\theta_{k}^{\prime}\right)} d+\sum_{k=0}^{N-1} n A_{k}^{\prime} e^{-j \theta_{k}^{\prime}}
$$

where $(\bullet)^{*}$ denotes the conjugate operation and $n$ is the additive white gaussian noise.

According to (5), it can be deduced that during the receiving process, the synchronization of the basic function is the most critical stage. When the spectrum at the transmitter and the receiver matches with each other, the basic functions shall be synchronized, which means $A_{k}=A^{\prime}{ }_{k}$ and $\theta_{k}=\theta^{\prime}{ }_{k}$. Thus the received signal at the receiver can be denoted as:

$$
y=\sum_{k=0}^{N-1} A_{k}^{2} d+\sum_{k=0}^{N-1} n A^{\prime}{ }_{k} e^{-j \theta_{k}^{\prime}}
$$

then the information can be received successfully. However, the mismatching in spectrum shall result to $A_{k} \neq A^{\prime}{ }_{k}$ and $\theta_{k} \neq \theta^{\prime}{ }_{k}$, hence the received signal is affected and become:

$$
y=\sum_{k=0}^{N-1} A_{k} A_{k}^{\prime} e^{j\left(\theta_{k}-\theta_{k}^{\prime}\right)} d+\sum_{k=0}^{N-1} n A_{k}^{\prime} e^{-j \theta_{k}^{\prime}}
$$

According to (7), the difference between $A_{k}$ and $A^{\prime}{ }_{k}$ will reduce the amplitude of the received signal to a low level, meanwhile the distinction in $\theta_{k}$ will result in phase distortion, both of which shall surely influence the performance of the system. However, the matching spectrum can only be available when the spectrum environment is almost the same at the transmitter and the receiver. Since such condition cannot be satistified easily (e.g. the communication distance is huge), some methods are required to deal with this problem. 


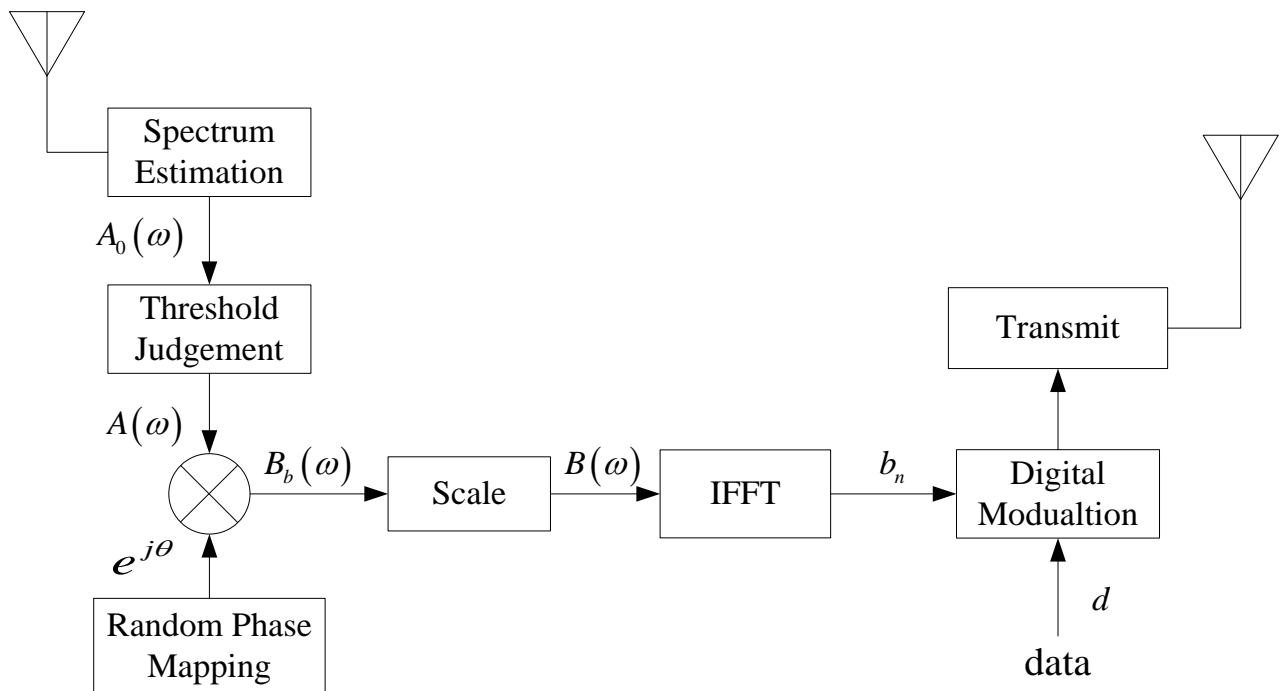

a

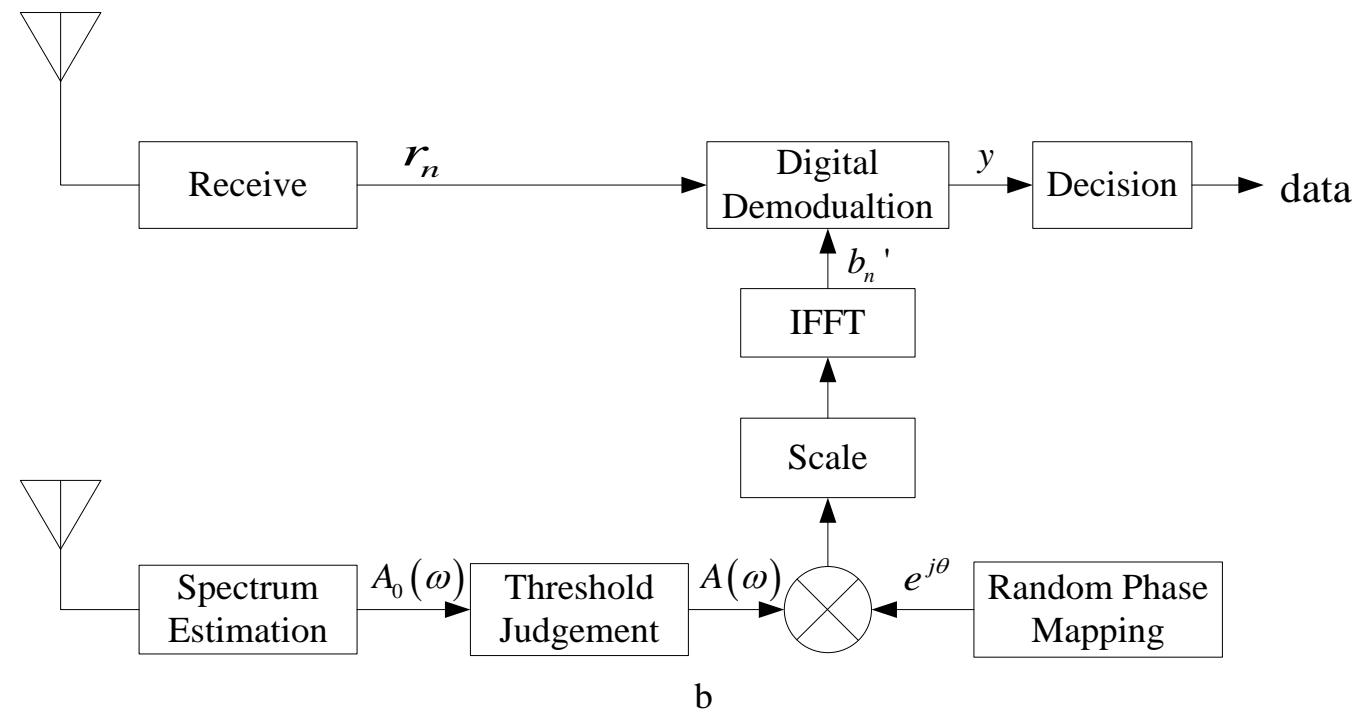

Fig. 1. Block diagram of traditional TDCS transmitter and receiver

\section{Modified Weighted Fractional Fourier Transform}

WFRFT is a generalized fourier transform which can be denoted as the weighted sum of different order fourier transforms of a signal [19]. The basic form of WFRFT is the 4-WFRFT, it can be extended to M-WFRFT (multiterm-WFRFT) and MP-WFRFT (multiple parameter WFRFT). 4-WFRFT is denoted as:

$$
\mathcal{F}^{\alpha}[\boldsymbol{X}]=\left(\omega_{0}^{\alpha} \boldsymbol{I}+\omega_{1}^{\alpha} \boldsymbol{F}+\omega_{2}^{\alpha} \boldsymbol{F}^{2}+\omega_{3}^{\alpha} \boldsymbol{F}^{3}\right) \boldsymbol{X}
$$


where $\boldsymbol{X}$ represents the original signal with length $N, \boldsymbol{I}$ is a $N \times N$ identity matrix, $\omega_{l}^{\alpha}(l=0,1,2,3)$ are the weight factors denoted as:

$$
\omega_{l}^{\alpha}=\frac{1}{4} \sum_{k=0}^{3} \exp \left[\frac{j 2 \pi(\alpha-l) k}{4}\right](l=0,1,2,3)
$$

$\boldsymbol{F}$ is the unitary discrete fourier transform matrix with the form of:

$$
\boldsymbol{F}_{m k}=(1 / N) \cdot \exp (-j 2 \pi(m-1)(k-1) / N)
$$

$\boldsymbol{F}^{2}$ and $\boldsymbol{F}^{3}$ are the second and third time of DFT operation respectively. Based on the symmetry of DFT, these two matrixes can be replaced by $\boldsymbol{P}$ and $\boldsymbol{P F} . \boldsymbol{P}$ is a $N \times N$ permutation matrix satisfying $P_{m, k}=\delta(\bmod (m+k, N))$, in which $\bmod (\cdot, \cdot)$ represents the modulo-N calculation [16]. In general, WFRFT can be simply denoted as:

$$
\mathcal{F}^{\alpha}[\boldsymbol{X}]=\boldsymbol{W}^{\alpha} \boldsymbol{X}^{T}
$$

where $\boldsymbol{W}^{\alpha}=\left[\omega_{0}^{\alpha} \boldsymbol{I}, \omega_{1}^{\alpha} \boldsymbol{F}, \omega_{2}^{\alpha} \boldsymbol{P I}, \omega_{3}^{\alpha} \boldsymbol{P F}\right]$ is a $N \times 4 N$ WFRFT matrix, $\boldsymbol{X}=\left[\boldsymbol{X}_{0}, \boldsymbol{X}_{0}, \boldsymbol{X}_{0}, \boldsymbol{X}_{0}\right]$ is an $1 \times 4 N$ vector, $\boldsymbol{X}_{0}$ is the original $1 \times N$ signal. So the result of 4 -WFRFT is a $N \times 1$ vector.

The WFRFT has numerous special characters, its application can be extended by making some modification. While transposing the $\boldsymbol{W}^{\alpha}$ and changing $\boldsymbol{X}$ to $\boldsymbol{X}^{\prime}=\left[\boldsymbol{X}_{0}^{T}, \boldsymbol{X}_{0}^{T}, \boldsymbol{X}_{0}^{T}, \boldsymbol{X}_{0}^{T}\right]$, we shall turn (11) to:

$$
\mathcal{F}_{\text {Mod }}^{\alpha}\left[\boldsymbol{X}^{\prime}\right]=\left(\boldsymbol{W}^{\alpha}\right)^{T} \boldsymbol{X}^{\prime}
$$

The result of (12) is a $4 N \times 4$ matrix, whose form is:

$$
\left[\begin{array}{cccc}
\omega_{0}^{\alpha} \boldsymbol{I} \boldsymbol{X}_{0}^{T} & \omega_{0}^{\alpha} \boldsymbol{I} \boldsymbol{X}_{0}^{T} & \omega_{0}^{\alpha} \boldsymbol{I} \boldsymbol{X}_{0}^{T} & \omega_{0}^{\alpha} \boldsymbol{I} \boldsymbol{X}_{0}^{T} \\
\omega_{1}^{\alpha} \boldsymbol{F} \boldsymbol{X}_{0}^{T} & \omega_{1}^{\alpha} \boldsymbol{F} \boldsymbol{X}_{0}^{T} & \omega_{1}^{\alpha} \boldsymbol{F} \boldsymbol{X}_{0}^{T} & \omega_{1}^{\alpha} \boldsymbol{F} \boldsymbol{X}_{0}^{T} \\
\omega_{2}^{\alpha} \boldsymbol{P} \boldsymbol{X}_{0}^{T} & \omega_{2}^{\alpha} \boldsymbol{P} \boldsymbol{X}_{0}^{T} & \omega_{2}^{\alpha} \boldsymbol{P} \boldsymbol{X}_{0}^{T} & \omega_{2}^{\alpha} \boldsymbol{P} \boldsymbol{X}_{0}^{T} \\
\omega_{3}^{\alpha} \boldsymbol{P} \boldsymbol{F} \boldsymbol{X}_{0}^{T} & \omega_{3}^{\alpha} \boldsymbol{P} \boldsymbol{F} \boldsymbol{X}_{0}^{T} & \omega_{3}^{\alpha} \boldsymbol{P} \boldsymbol{F} \boldsymbol{X}_{0}^{T} & \omega_{3}^{\alpha} \boldsymbol{P} \boldsymbol{F} \boldsymbol{X}_{0}^{T}
\end{array}\right]
$$

It is noteworthy that the sum of each column is the WFRFT to $\boldsymbol{X}_{0}$, bespeaking that four WFRFT signals can be acquired together after the modification. Thus by replacing $\boldsymbol{X}_{0}^{T}$ in $\boldsymbol{X}^{\prime}$ to other signals like $\boldsymbol{X}_{1}^{T}, \boldsymbol{X}_{2}^{T}, \boldsymbol{X}_{3}^{T}$ and $\boldsymbol{X}_{4}^{T}$, (13) shall be turned to:

$$
\left[\begin{array}{cccc}
\omega_{0}^{\alpha} \boldsymbol{I} \boldsymbol{X}_{1}^{T} & \omega_{0}^{\alpha} \boldsymbol{I} \boldsymbol{X}_{1}^{T} & \omega_{0}^{\alpha} \boldsymbol{I X}_{1}^{T} & \omega_{0}^{\alpha} \boldsymbol{I} \boldsymbol{X}_{1}^{T} \\
\omega_{1}^{\alpha} \boldsymbol{F} \boldsymbol{X}_{2}^{T} & \omega_{1}^{\alpha} \boldsymbol{F} \boldsymbol{X}_{2}^{T} & \omega_{1}^{\alpha} \boldsymbol{F} \boldsymbol{X}_{2}^{T} & \omega_{1}^{\alpha} \boldsymbol{F} \boldsymbol{X}_{2}^{T} \\
\omega_{2}^{\alpha} \boldsymbol{P} \boldsymbol{X}_{3}^{T} & \omega_{2}^{\alpha} \boldsymbol{P} \boldsymbol{X}_{3}^{T} & \omega_{2}^{\alpha} \boldsymbol{P} \boldsymbol{X}_{3}^{T} & \omega_{2}^{\alpha} \boldsymbol{P} \boldsymbol{X}_{3}^{T} \\
\omega_{3}^{\alpha} \boldsymbol{P} \boldsymbol{F} \boldsymbol{X}_{4}^{T} & \omega_{3}^{\alpha} \boldsymbol{P} \boldsymbol{F} \boldsymbol{X}_{4}^{T} & \omega_{3}^{\alpha} \boldsymbol{P} \boldsymbol{F} \boldsymbol{X}_{4}^{T} & \omega_{3}^{\alpha} \boldsymbol{P} \boldsymbol{F} \boldsymbol{X}_{4}^{T}
\end{array}\right]
$$


In the wake of summing each column of the matrix above, we shall eventually acquire:

$$
\left[\mathcal{F}\left[\boldsymbol{X}_{1}\right], \mathcal{F}\left[\boldsymbol{X}_{2}\right], \mathcal{F}\left[\boldsymbol{X}_{3}\right], \mathcal{F}\left[\boldsymbol{X}_{4}\right]\right]
$$

which means the modified WFRFT can transmit different signals simultaneously. These signals could be different useful signals, the local spectral information, artificial noises and other distinctive combinations. With this modification, the application of WFRFT shall be extended to wilder area.

\section{System Description and Theoretical Performance}

The proposed modified WFRFT-based system is illustrated in Fig. 2. At the transmitter, the spectral vector $B(\omega)$ and the modulated signals are incorporated to form a new signal matrix. On that basis this signal matrix is transmitted to the modified WFRFT block to output the transmitting signal. At the receiver, the $-\alpha$ order WFRFT is done every $N$ symbols of the received signal to recover the spectral vector and the modulated signals for demodulation. Since this paper lays particular stress on the performance analysis over spectrum mismatching condition, the fading resist procedures, e.g. equalization, have been omitted in Fig. 2 for clarity. The idea of the proposed system is transmitting the spectrum information for the basic function with the TDCS signals by taking advantage of the modified WFRFT to solve the problem when applying TDCS under spectrum mismatching condition.

\subsection{Transmitter}

As it is indicated in Fig. 2, the TDCS detects the occupied spectrum of the current environment and acquires the available spectrum by setting the amplitudes of the occupied frequency components at zero. The multi-valued complex pseudorandom phase vector $e^{j \theta}$ is multiplied element by element with $A^{\prime}(\omega)$ to generate the spectral vector $B(\omega)$. In the wake of the IFFT operation, the basic function $b_{n}$ is obtained, which is subsequently stored for modulation. On that basis the modulated signals $\boldsymbol{X}_{i}(i=1,2,3)$ and the spectrum vector $B(\omega)$ are transmitted to the signal combination block to output the new signal matrix $\boldsymbol{X}^{\prime}=\left[\begin{array}{llll}\boldsymbol{B}^{T} & \boldsymbol{X}_{1}^{T} & \boldsymbol{X}_{2}^{T} & \boldsymbol{X}_{3}^{T}\end{array}\right]$. An $\alpha$ order modified WFRFT is done to $\boldsymbol{X}^{\prime}$ in the modified WFRFT block, whose output is: 


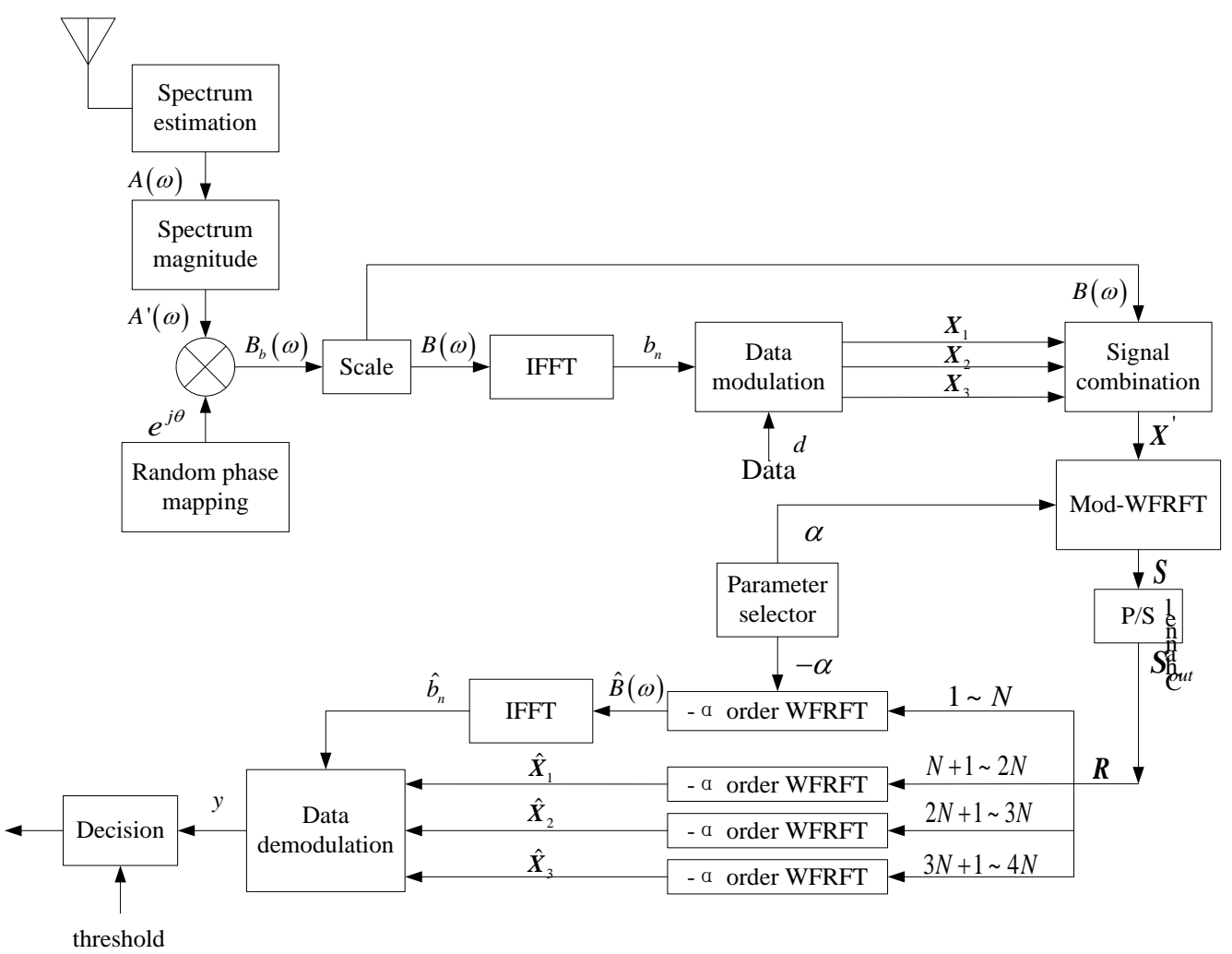

Fig. 2. System block diagram of the modified WFRFT-based TDCS. The spectral vector and the modulated signals are combined to a new signal matrix and transmitted to the modified WFRFT block at the transmitter. At the receiver, the $-\alpha$ order WFRFT is done column-wise to the matrix to recover the

$$
\boldsymbol{S}=\mathcal{F}_{\text {Mod }}^{\alpha}\left[\boldsymbol{X}^{\prime}\right]=\left[\begin{array}{llll}
\sum_{0}^{3} \omega_{l}^{\alpha} \boldsymbol{F}_{N}^{l} \boldsymbol{B}^{T} & \sum_{0}^{3} \omega_{l}^{\alpha} \boldsymbol{F}_{N}^{l} \boldsymbol{X}_{1}^{T} & \sum_{0}^{3} \omega_{l}^{\alpha} \boldsymbol{F}_{N}^{l} \boldsymbol{X}_{2}^{T} & \sum_{0}^{3} \omega_{l}^{\alpha} \boldsymbol{F}_{N}^{l} \boldsymbol{X}_{3}^{T}
\end{array}\right]
$$

where $\boldsymbol{F}_{N}$ is the N-points DFT matrix, $\boldsymbol{B}^{T}$ and $\boldsymbol{X}_{i}(i=1,2,3)$ are $N \times 1$ vectors. $\boldsymbol{S}$ is a $N \times 4$ matrix. Moreover, the signal $\boldsymbol{S}_{\text {out }}$ in Fig. 2 is an $1 \times 4 N$ vector whose elements are taken column-wise from $\boldsymbol{S}$.

\subsection{Receiver}

When transmitting through the channel, the communication signal received can be defined as:

$$
\boldsymbol{R}=\boldsymbol{H} \boldsymbol{S}_{\text {out }}+\boldsymbol{n}_{0}
$$

where $\boldsymbol{H}$ denotes the channel fading matrix, for the channel acting as an AWGN channel, $\boldsymbol{H}$ represents an identity matrix. $\boldsymbol{n}_{0}$ is the AWGN noise with the variance $\sigma_{n}^{2}$.

When the signal arrives at the legitimate receiver, it is separated into four signal vectors every $N$ symbols. These four signals are transmitted to four $-\alpha$ order WFRFT block to recover the spectrum information $\hat{\boldsymbol{B}}$ and the TDCS signals $\hat{\boldsymbol{X}}_{i}(i=1,2,3)$. As the WFRFT can be denoted as equation (11), $\hat{\boldsymbol{B}}$ and $\hat{\boldsymbol{X}}_{i}(i=1,2,3)$ are denoted as: 


$$
\begin{gathered}
\hat{\boldsymbol{B}}=\boldsymbol{W}^{-\alpha} \boldsymbol{R}_{0 N}^{T} \\
\hat{\boldsymbol{X}}_{i}=\boldsymbol{W}^{-\alpha} \boldsymbol{R}_{i N}^{T}(i=1,2,3)
\end{gathered}
$$

where $\boldsymbol{W}^{-\alpha}$ is the $-\alpha$ WFRFT matrix, $\boldsymbol{R}_{i N}$ refers to the $i \times N+1$ th symbol to $(i+1) \times N$ th symbol of $\boldsymbol{R}$. Then the basic function can be recovered from $\hat{\boldsymbol{B}}$ after an IFFT block to demodulate $\hat{\boldsymbol{X}}_{i}(i=1,2,3)$.

\subsection{Theoretical Performance of the System}

To demonstrate the performance of the proposed system, it is necessary to derive its theoretical BER. By substituting (12) into (17), the received signal vector can be denoted as:

$$
\boldsymbol{R}=\left(\boldsymbol{W}^{\alpha}\right)^{T} \boldsymbol{H} \boldsymbol{X}^{\prime}+\boldsymbol{n}_{0}
$$

According to (11) and (16), $\boldsymbol{R}_{i N}$ is denoted as:

$$
\boldsymbol{R}_{i N}=\left(\boldsymbol{W}^{\alpha} \boldsymbol{H}^{T} \boldsymbol{X}_{i}^{T}\right)^{T}+\boldsymbol{n}_{0}(i=1,2,3)
$$

By substituting (21) into (19), the $\hat{\boldsymbol{X}}_{i}$ shall be denoted as:

$$
\hat{\boldsymbol{X}}_{i}=\boldsymbol{W}^{-\alpha}\left(\left(\boldsymbol{W}^{\alpha} \boldsymbol{H}^{T} \boldsymbol{X}_{i}^{T}\right)^{T}+\boldsymbol{n}_{0}\right)^{T}=\boldsymbol{W}^{-\alpha}\left(\boldsymbol{W}^{\alpha} \boldsymbol{H}^{T} \boldsymbol{X}_{i}^{T}+\boldsymbol{n}_{0}^{T}\right)=\boldsymbol{H}^{T} \boldsymbol{X}_{i}^{T}+\boldsymbol{W}^{-\alpha} \boldsymbol{n}_{0}^{T} \quad(i=1,2,3)
$$

Assuming $\boldsymbol{W}^{-\alpha} \boldsymbol{n}_{0}^{T}=\boldsymbol{n}$ and the mean and variance of it is zero and $\sigma^{2}$ respectively, the $\hat{\boldsymbol{X}}_{i}(i=1,2,3)$ is thereupon a gaussian signal that obeys the distribution $N\left(0, \sigma^{2}\right)$. In this regard, the theoretical BER of the proposed system is:

$$
\begin{gathered}
B E R_{A W G N}=Q\left(\sqrt{\frac{E_{s}(1-\rho)}{N_{b}}}\right) \\
B E R_{\text {Rayleigh, } Q P S K}=2 Q\left(\sqrt{\frac{E_{s}}{N_{b}}}\right)\left[1-\frac{1}{2} Q\left(\sqrt{\frac{E_{s}}{N_{b}}}\right)\right]
\end{gathered}
$$

where $E_{s}$ refers to the energy per symbol of the signal, $N_{b}$ represents the power of $\boldsymbol{n}$, and $\rho$ is the cross-correlation coefficient. To antipodal signals, $\rho=-1$ and to orthogonal signals, $\rho=0 . Q(\bullet)$ is the co-error function with the form of:

$$
Q(x) \approx \frac{1}{\sqrt{2 \pi}} \int_{x}^{\infty} \exp \left(-\frac{u^{2}}{2}\right) d u
$$

\section{Simulation results and discussion}

The reliability of a communication system is commonly evaluated by the BER. The scenario is established as follows: the original information is modulated by bipolar modulation, cyclic shift keying modulation and cyclic antipodal shift keying modulation and be jointly transmitted by the system. The parameter $\alpha$ is set to 0.8 . Since 0.5 means totally fractional and 1 means traditional FFT, such a parametere between 0.5 and 1 can show the characteristic of the modified WFRFT better. Fig. 3 indicates the system BER as different modulated signals 
are being transmitted. It can be deduced from the simulation results that the BER of the different signals are all fitted with the theoretical value, bespeaking that the proposed system performs well in reliability. The different signals transmitted together shall not affect each other. Another performance required to prove is the system reliability when the spectrum is different at the transmitter and the receiver. The spectrum difference is established at $0,40 \%$ and $80 \%$ to test the reliability performance of the proposed system and compares it with the traditional TDCS. During the simulation, the spectrum consists of different frequency points. According to the difference percent, several points are changed at the receiver to simulate the spectrum difference. Fig. 4 shows the results of the simulation. It can be inferred that in the presence of 0 spectrum difference, the traditional TDCS outperforms the proposed system. However, when the difference in spectrum increasing, the performance of the traditional TDCS is negatively affected and worsen, while the performance of the proposed system remains steady as the spectrum information being transmitted in the signal. We also compare the performance of the proposed system with the method proposed in [12]. The results are presented in Fig. 5. As the simulation results indicate, the BER performance of the method in [12] outstrips that of the proposed system, but the precondition is that the phase information has been already known by the receiver, which is not possible in some conditions. For instance, in the presence of $20 \%$ mismatching in the phase information, the method in [12] takes on worse performance than the proposed system. With the increasing of SNR, the BER performance of the proposed system without phase information is closing to the method in [12] with full phase information, bespeaking that the applicable environment of the proposed system is much wilder without losing much BER performance.

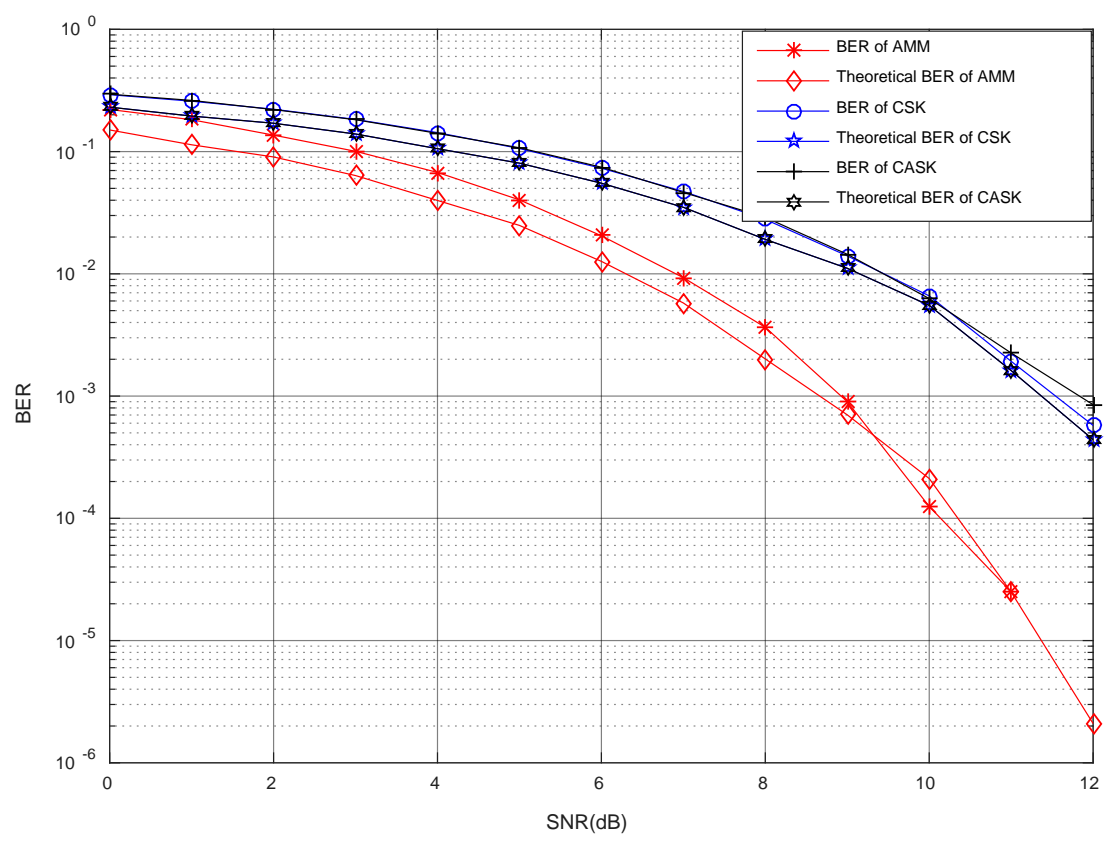

Fig. 3. Simulation results over AWGN channel for the proposed system through evaluating the BER performance with different modulation methods for $\alpha=0.8$. The three kinds of modulation methods fit the theoretical value, bespeaking that the reliability of the system is independent of its modification. 
The performance of this system is also simulated under multi-path fading channel environment. The results are exhibited in Fig. 6. The simulation environment is set as follow: The number of path is 6 , each of them suffers a time delay of $0.5-1$ second. The doppler frequency is set as $200 \mathrm{~Hz}$. The fading channels are simulated as Rayleigh fading channels. According to the Figure, it is noteworthy that the BER performance of the modified WFRFT-based TDCS is in the vicinity of the theoretical result, bespeaking that the modified WFRFT prevents the signals and the basic function from suffering the multi-path fading. Furthermore, the traditional TDCS is required to transmit the basic function to the receiver, and yet it shall be affected by the frequency selective fading channel. The demodulation at the receiver shall be negatively affected consequently. As a result, the BER performance of traditional TDCS is not adequately available to receive information from the signal.

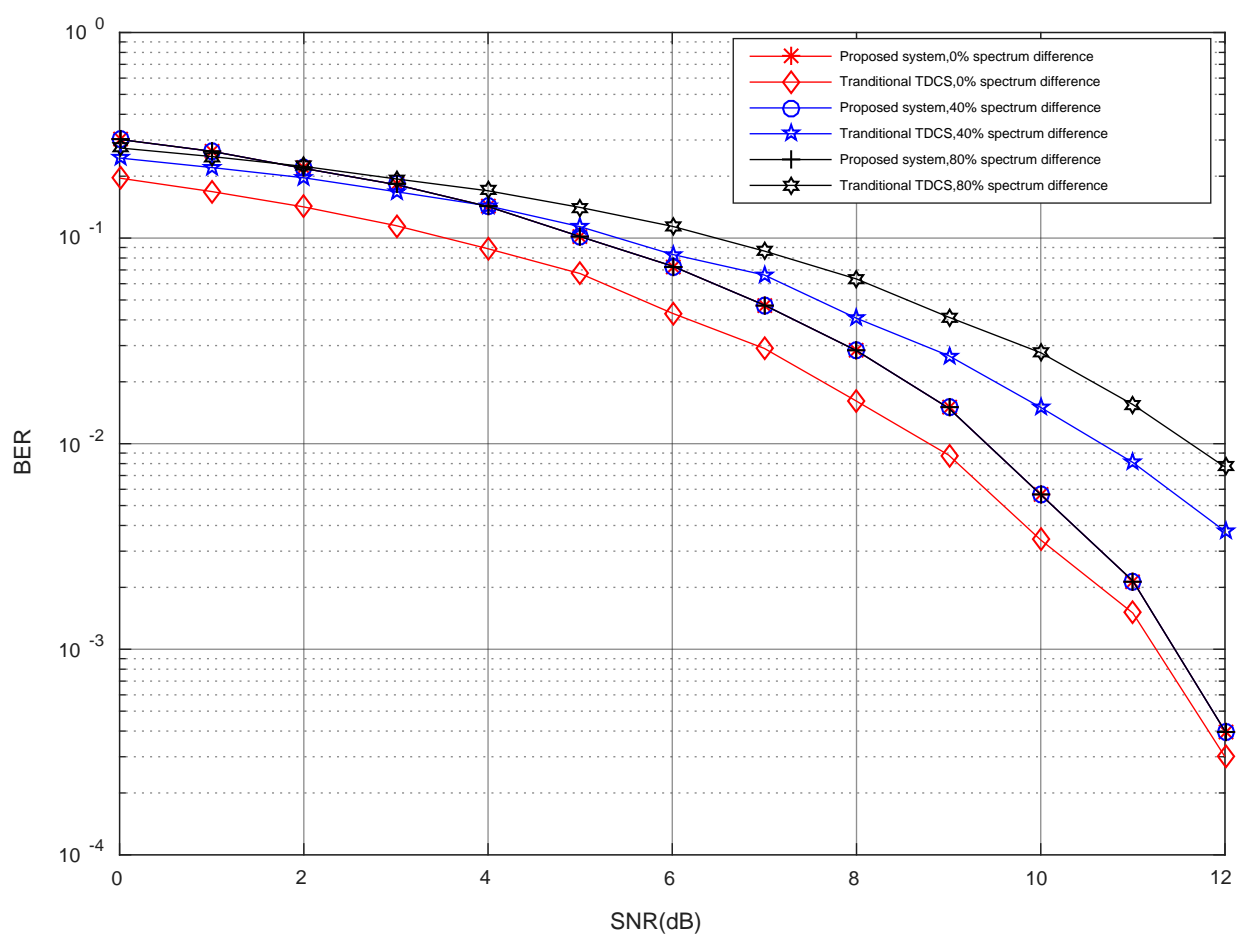

Fig. 4. Simulation results on AWGN channel for the proposed system by evaluating the BER performance with spectrum difference of $0,40 \%$ and $80 \%$. The performance of the traditional TDCS is negatively affected and worsen, while the performance of the proposed system remains steady as the difference in spectrum increasing. 


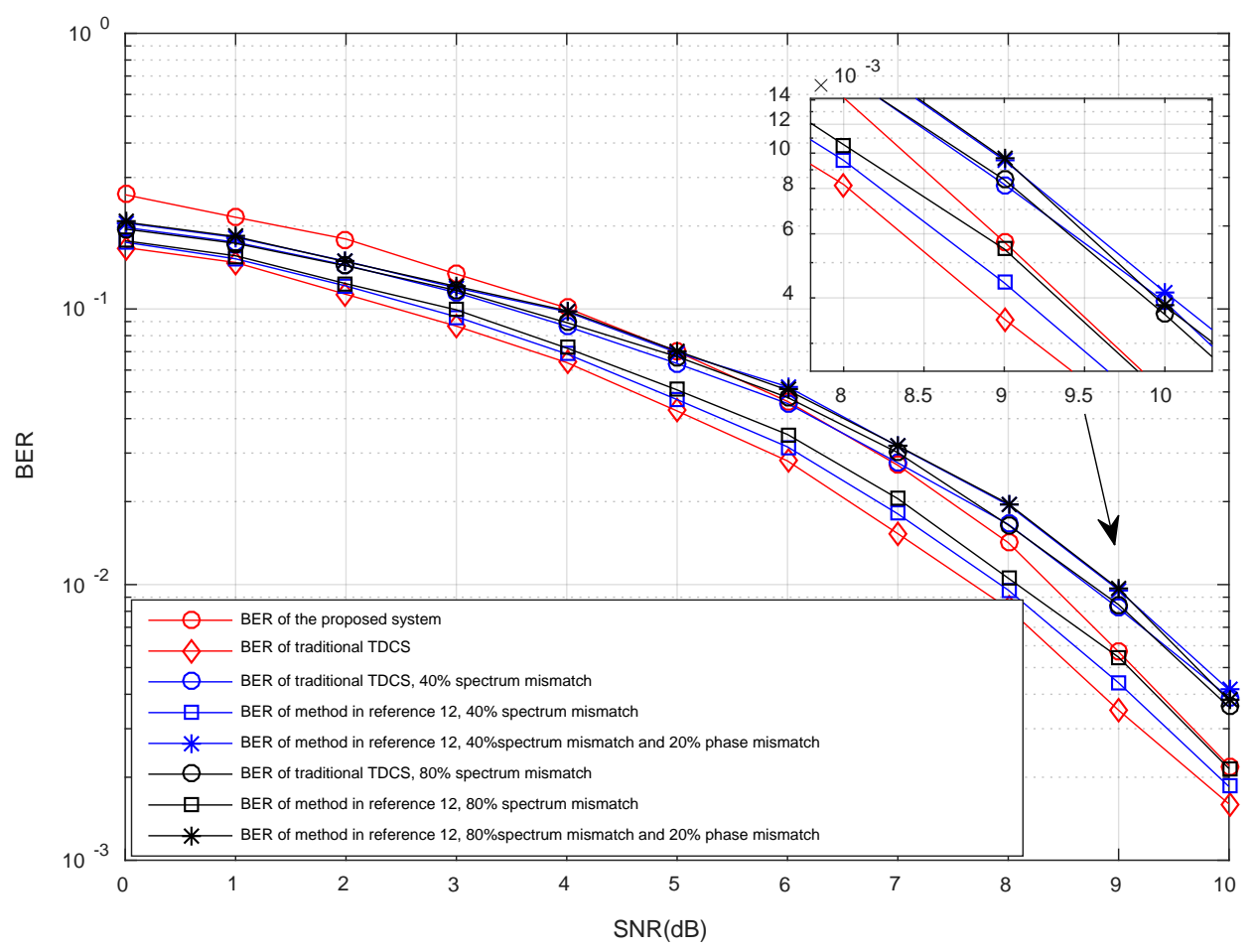

Fig. 5. BER performance comparison between the proposed system and the method in reference [12]. The BER performance of the method in [12] outstrips the proposed system when the phase information is attained by the receiver. Yet in the presense of $20 \%$ mismatching in phase information, the BER of the method in [12] decreases while the BER of the proposed system remains steady.

To ascertain the built-in security property of the proposed system, the following scenario is factored in. The eavesdropper is hypothesized to be unaware of the parameter $\alpha$, though some communication parameters have been known publically (inclusive of the sample rate, etc.). So it is reasonable to ascertain the secrecy of the proposed system by analyzing the BER performance of received signal at the eavesdropper. The anti-eavesdrop performance of the proposed system is presented in Fig. 7 and Fig. 8. Fig. 7 demonstrates the constellation of the traditional TDCS signal and the signal of proposed system with different parameter. It indicates that the constellation of traditional TDCS signal has been dispersed. The greater $\alpha$ causes greater dispersion and when $\alpha=0.8$, the core of the constellation starts to spread out. This shall make the eavesdropper more difficult to intercept the signal by constellation recognition.

Fig. 8 draws the comparison between the BER performance of the proposed system and the traditional TDCS at the eavesdropper. It is hypothesized here that the basic function is acquired by the eavesdropper, which is possible if it is transmitted by the public channel. It can be attained from Fig. 8 that the traditional TDCS signal can be intercepted by the eavesdropper whereas the signal transmitted by the proposed system can still keep the information secured with the protection of the modified WFRFT. 


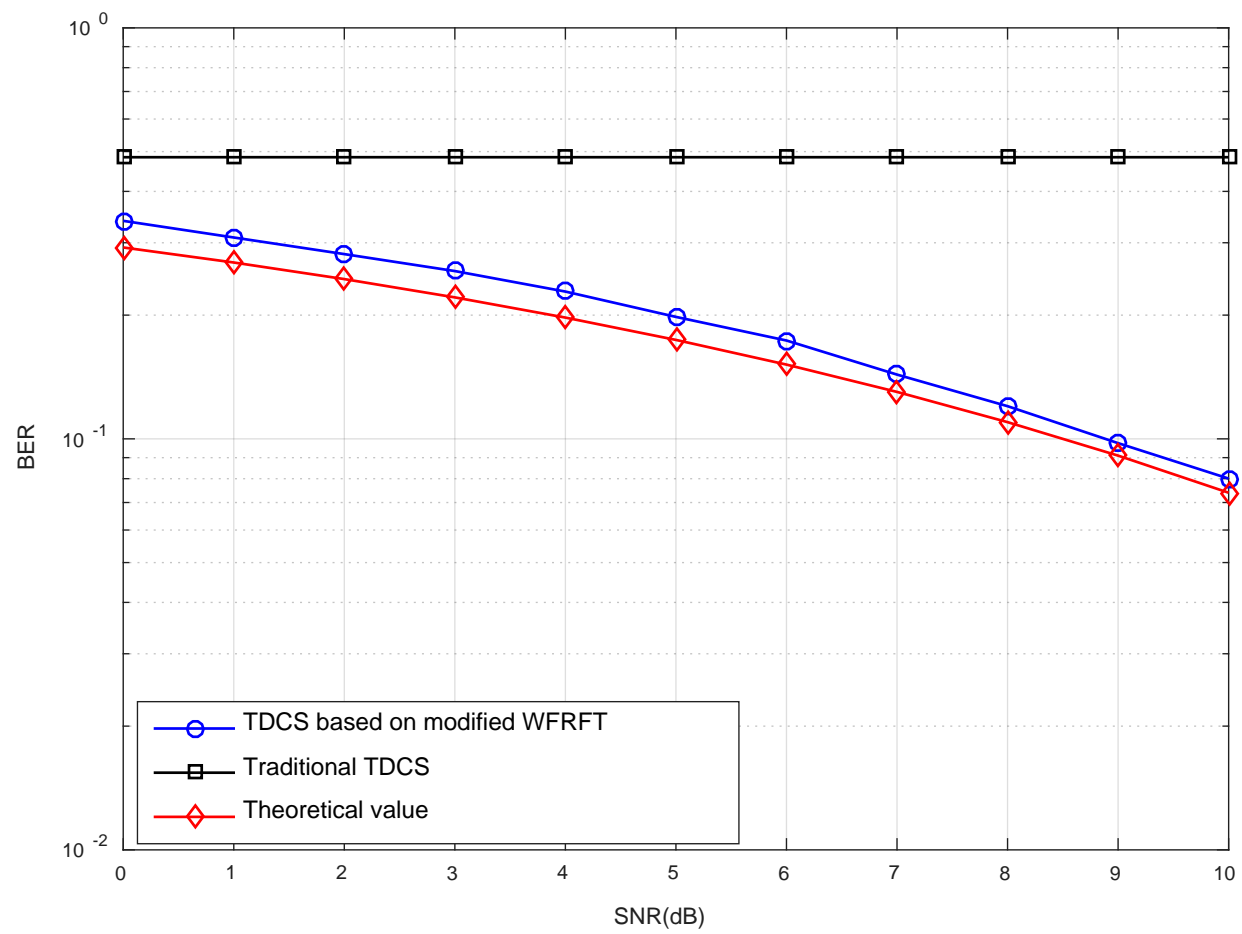

Fig. 6. BER performance comparison between the proposed system and the traditional TDCS. The performance of the proposed system is much better than the traditional TDCS system as the transmitted signal of it is protected by the modified WFRFT.

\section{Conclusion}

In this paper, a modified WFRFT-based TDCS is proposed to solve the spectrum mismatching and enhance the security of traditional TDCS. The basic function can be transmitted jointly with the modulated signals through modifying the WFRFT and adopting it into the TDCS. At the receiver, an $-\alpha$ order WFRFT is conducted to the received signal, and accordingly the basic function is attained to demodulate the signals. As the simulation results indicate, the BER performances can be well geared into the theoretical analysis. When the transmitter and the receiver are different in spectrum, the proposed system shall remain reliable. Furthermore, the constellation of traditional TDCS signal is dispersed by the WFRFT. As the necessity of transmitting basic function in public channel is avoided, the security of signal is enhanced. In general, the proposed system intensify the traditional TDCS whereby a modified WFRFT, which makes the signal transmission more flexible and optimizes the security. 

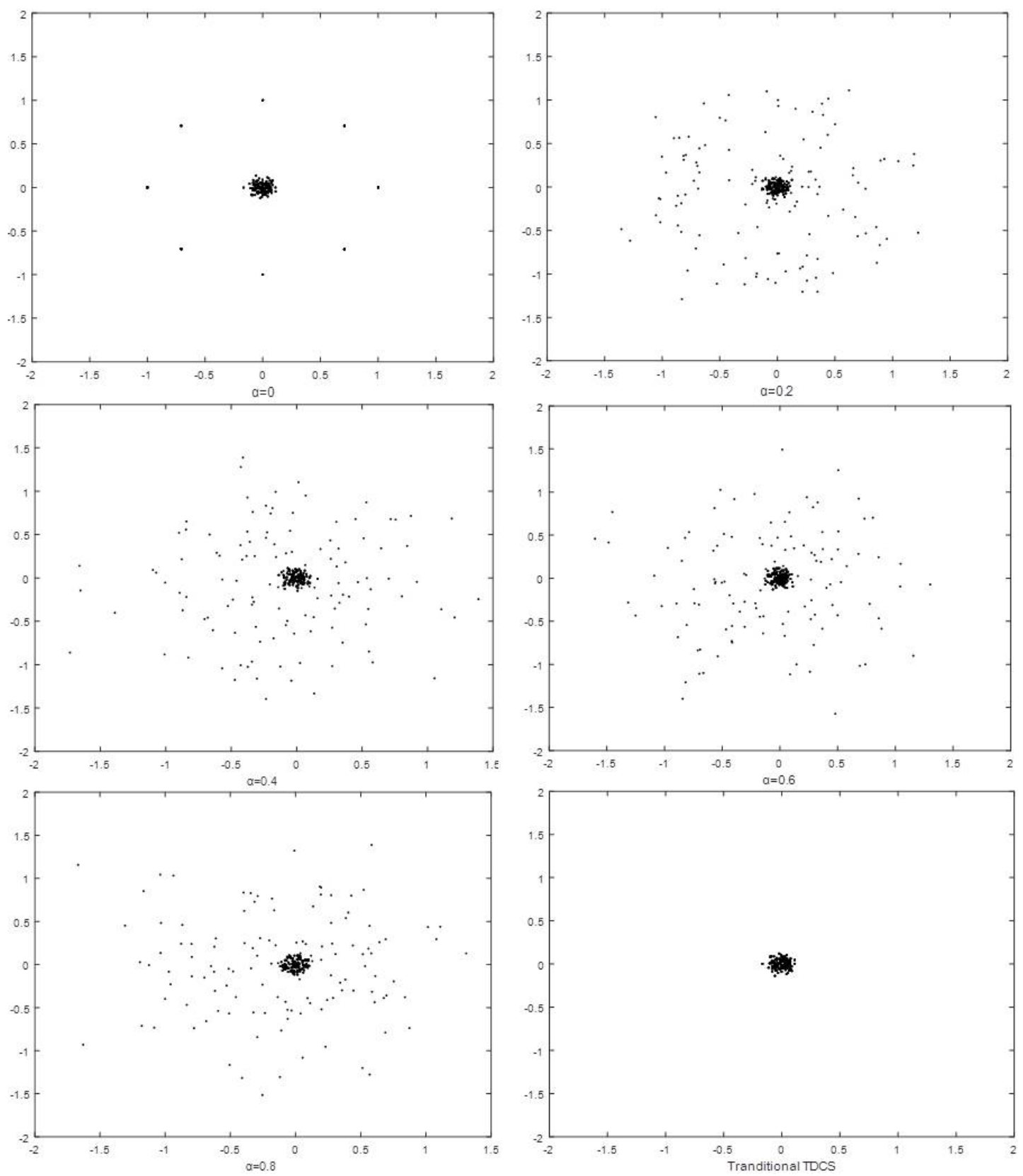

Fig. 7. The constellation of the signal with different parameter $\alpha$. The greater $\alpha$ causes greater dispersion and when $\alpha=0.8$, the core of the constellation starts to spread out. 


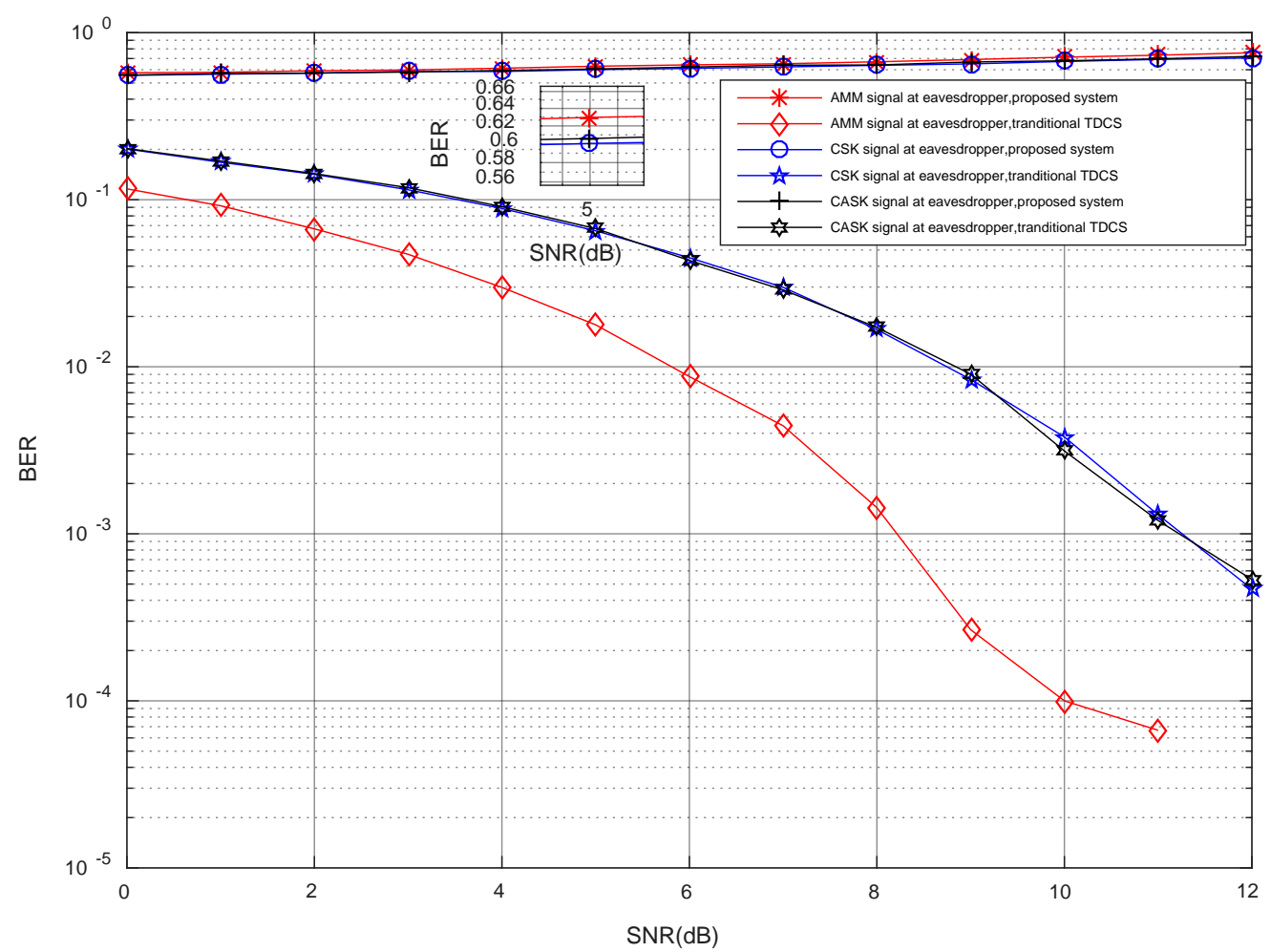

Fig. 8. Secrecy performance comparison of modified WFRFT-TDCS with traditional TDCS. The traditional TDCS signal can be intercepted by the eavesdropper whereas the signal transmitted by the proposed system can still keep the information secret with the protect of the modified WFRFT.

\section{References}

[1] T. S. Nguyen, C. C. Chang, and X. Q. Yang, “A reversible image authentication scheme based on fragile watermarking in discrete wavelet transform domain,” AEU - International Journal of Electronics and Communications, vol. 70, no. 8, pp. 1055-1061, 2016. Article (CrossRef Link)

[2] O. S. Faragallah, "Efficient video watermarking based on singular value decomposition in the discrete wavelet transform domain,” $A E U$ - International Journal of Electronics and Communications, vol. 67, no. 3, pp. 189-196, 2013. Article (CrossRef Link)

[3] S. Hu, G. Bi, Y. L. Guan et al., "TDCS-Based Cognitive Radio Networks with Multiuser Interference Avoidance,” IEEE Transactions on Communications, vol. 61, no. 12, pp. 4828-4835, 2013. Article (CrossRef Link)

[4] G. Wu, S. Hu, and S. Li, "Low complexity time-frequency synchronization for transform domain communications systems." IEEE China Summit and International Conference on Signal and Information Processing, pp. 1002-1006. Article (CrossRef Link)

[5] S. Wang, X. Da, Z. Chu et al., "Magnitude weighting selection: a method for peak-to-average power ratio reduction in transform domain communication system,” Communications Iet, vol. 9, no. 15, pp. 1894-1901, 2015. Article (CrossRef Link)

[6] C. Chang, H. Huan, J. Xu et al., "Multidimensional parallel combinatory transform domain communication system,” International Journal of Communication Systems, pp. e3249, 2017. Article (CrossRef Link)

[7] R. Lin, G. Bi, X. Liu et al., "On the modulation and signalling design for a transform domain communication system,” Communications Iet, vol. 8, no. 16, pp. 2909-2916, 2014.

Article (CrossRef Link) 
[8] N. Q. Wei, and Z. L. Chen, "A Design of Basis Function for Transform Domain Communication System," Applied Mechanics and Materials, vol. 347-350, pp. 2143-2147, 2013. Article (CrossRef Link)

[9] Y. Liang, X. Da, W. An et al., "Optimal design of double-threshold basic function in transform domain communication system,” XI Tong Gong Cheng Yu Dian Zi Ji Shu/systems Engineering \& Electronics, pp. 634-640, 2017. Article (CrossRef Link)

[10] S. P. Ni, and L. H. Deng, "Application on OFDM-Based Transform Domain Communication System in Mine,” Applied Mechanics and Materials, vol. 143-144, pp. 843-848, 2011. Article (CrossRef Link)

[11] G. Fumat, P. Charge, A. Zoubir et al., "Enhancing spectral efficiency of transform domain communication systems by using a multidimensional modulation," in Proc. of International ICST Conference on Cognitive Radio Oriented Wireless Networks and Communications, pp. 131-135, 2011. Article (CrossRef Link)

[12] T. C. Xie, X. Y. Da, Z. Y. Chu et al., "Basis function design for transform domain communication system in the presence of spectral mismatches," Jilin Daxue Xuebao, vol. 44, no. 6, pp. 1825-1830, 2014. Article (CrossRef Link)

[13] N. Goel, and K. Singh, "Convolution and correlation theorems for the offset fractional Fourier transform and its application," AEU - International Journal of Electronics and Communications, vol. 70, no. 2, pp. 138-150, 2016. Article (CrossRef Link)

[14] L. Mei, X. Sha, Q. Ran et al., "Research on the application of 4-weighted fractional Fourier transform in communication system," Science China Information Sciences, vol. 53, no. 6, pp. 1251-1260, 2010. Article (CrossRef Link)

[15] L. Mei, X. J. Sha, and N. T. Zhang, "The approach to carrier scheme convergence based on 4-weighted fractional fourier transform," IEEE Communications Letters, vol. 14, no. 6, pp. 503-505, 2010. Article (CrossRef Link)

[16] K. Wang, X. Sha, L. Mei et al., "Performance Analysis of Hybrid Carrier System with MMSE Equalization over Doubly-Dispersive Channels," IEEE Communications Letters, vol. 16, no. 7, pp. 1048-1051, 2012. Article (CrossRef Link)

[17] K. Wang, X. Sha, and L. Mei, "On Interference Suppression in Doubly-Dispersive Channels with Hybrid Single-Multi Carrier Modulation and an MMSE Iterative Equalizer," IEEE Wireless Communications Letters, vol. 1, no. 5, pp. 504-507, 2012. Article (CrossRef Link)

[18] X. J. Sha, X. Qiu, and L. Mei, "Hybrid Carrier CDMA Communication System Based on Weighted-Type Fractional Fourier Transform,” IEEE Communications Letters, vol. 16, no. 4, pp. 432-435, 2012. Article (CrossRef Link)

[19] L. Mei, Q. Zhang, X. Sha et al., "WFRFT Precoding for Narrowband Interference Suppression in DFT-Based Block Transmission Systems," IEEE Communications Letters, vol. 17, no. 10, pp. 1916-1919, 2013. Article (CrossRef Link)

[20] L. Mei, Q. Zhang, X. Sha et al., "WFRFT Precoding for Narrowband Interference Suppression in DFT-Based Block Transmission Systems,” IEEE Communications Letters, vol. 17, no. 10, pp. 1916-1919, 2013. Article (CrossRef Link)

[21] Fang, Xiaojie, and Xuejun, "Guaranteeing Wireless Communication Secrecy via a WFRFT-Based Cooperative System,” China Communications, vol. 12, no. 9, pp. 76-82, 2015. Article (CrossRef Link)

[22] X. Fang, X. Sha, and Y. Li, "MP-WFRFT and constellation scrambling based physical layer security system,” China Communications, vol. 13, no. 2, pp. 138-145, 2016. Article (CrossRef Link)

[23] F. Xiaojie, S. Xuejun, and L. Yong, "Secret Communication Using Parallel Combinatory Spreading WFRFT,” Communications Letters IEEE, vol. 19, no. 1, pp. 62-65, 2015. Article (CrossRef Link)

[24] Z. Luo, H. Wang, and K. Zhou, "Physical Layer Security Scheme Based on Polarization Modulation and WFRFT Processing for Dual-polarized Satellite Systems," KSII TRANSACTIONS ON INTERNET AND INFORMATION SYSTEMS, vol. 11, pp. 5610-5624, 2017. Article (CrossRef Link) 
[25] Z. Luo, H. Wang, K. Zhou et al., “Combined Constellation Rotation with Weighted FRFT for Secure Transmission in Polarization Modulation Based Dual-polarized Satellite Communications,” IEEE Access, vol. PP, no. 99, pp. 1-1, 2017. Article (CrossRef Link)

[26] Z. Luo, H. Wang, and K. Zhou, "Polarization Filtering Based Physical-layer Secure Transmission Scheme for Dual-polarized Satellite Communication,” IEEE Access, vol. PP, no. 99, pp. 24706-24715, 2017. Article (CrossRef Link)

[27] P. J. Swackhammer, M. A. Temple, and R. A. Raines, "Performance simulation of a transform domain communication system for multiple access applications,” in Proc. of Military Communications Conference, pp. 1055-1059 vol.2. Article (CrossRef Link)

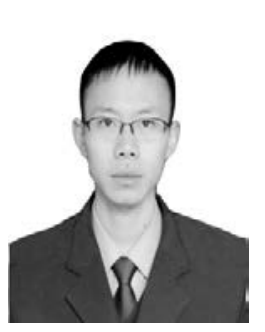

Ruiyang Xu received the B.Sc. degree in automatic control engineering and the M.A.Sc degree in information and communication engineering from Air Force Engineering University, China, in 2013, and 2016, respectively. He is currently pursuing his Ph.D. at Institute of Information and Navigation, Air Force Engineering University, Xi'an, China. His research interests include satellite communications, covert communications, optimization and intelligent algorithm. E-mail: lixueyuanxuruiyang@126.com

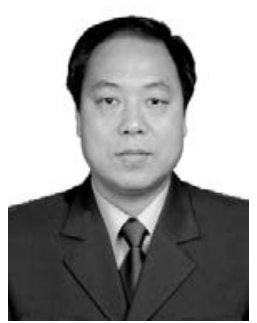

Xinyu Da received the B.Sc. in Xidian University, the M.A.Sc degree in communication and electronic system from Air and Missile Defense College, and the Ph.D. degree from School of Marine Science and Technology, NPU in 1983, 1988 and 2007 respectively. He is working as the professor in Information and Navigation College, AFEU. His research interests include Satellite communications, communication theory, signal processing, transform domain communication system and cognitive radio. E-mail: kgddxy2008@163.com

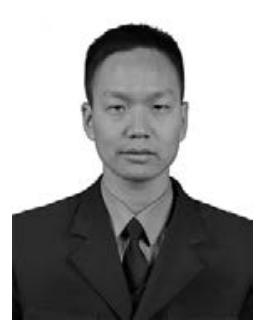

Yuan Liang received the B.Sc. degree in information and navigation engineering and the M.A.Sc degree in information and communication engineering from Air Force Engineering University, China, in 2012, and 2015, respectively. He is currently pursuing his Ph.D. at Institute of Information and Navigation, Air Force Engineering University, Xi'an, China. His research interests include intelligent signal processing, wireless communications, secure communications, optimization and intelligent algorithm. E-mail: lycrazy0925@163.com

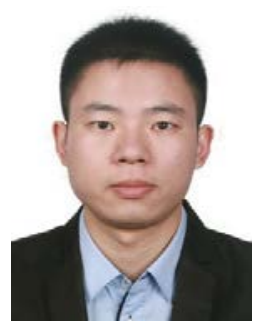

Hang Hu received the B. S. degree in Telecommunications Engineering from Xidian University, Xi'an, China in 2010, the M.S. degree and Ph. D degree in Information and Communications Engineering at College of Communications Engineering, PLA University of Science and Technology, Nanjing, China, in 2012 and 2016, respectively. He is now a lecturer at Information and Navigation College, Air Force Engineering University in Xi'an, China. He received the Grant of Postdoctoral Innovative Talent Program in China in 2017. His current research interests include cognitive radio technology, cooperative communications and green communications. E-mail: xd_huhang@126.com 